\title{
Multi-Dimensional Sport Physique Evaluation and Ranking for Healthcare Based on TOPSIS
}

\author{
Changqing Zhuo, The Engineering \& Technical College of Chengdu University of Technology, China \\ Qiyun Zhang, Shandong Provincial University Laboratory for Protected Horticulture, Weifang University of Science and \\ Technology, Shouguang, China \\ Qin Xie, Sichuan Staff University of Science and Technology, China \\ Yinping Lin, No. 1 Middle School of Pidu Chengdu, China \\ Chengxin Xu, School of Computer Science, Qufu Normal University, China \\ Lina Wang, School of Computer Science, Qufu Normal University, China \\ Sifeng Wang, School of Computer Science, Qufu Normal University, China
}

\begin{abstract}
Typically, through monitoring, collecting, integrating, and analyzing the sport scores of individuals, we can know about the sport physique of the individuals in a real-time manner, so as to further guarantee a high-quality health level of people. However, in the abovementioned sport scores-based sport physique monitoring scenario, two major challenges are raised. First, the collected sport scores for sport physique monitoring are often multi-dimensional, which makes it hard to evaluate the sport physique of individuals fairly and reasonably. Moreover, the multiple dimensions of sport scores are often of different weights or significances, which raise a challenging task to objectively evaluate the sport physique of an individual. Considering these challenges, a novel multi-dimensional sport physique evaluation and ranking method, named MSPER, is proposed in this research work for better healthcare for individuals, which is based on the TOPSIS theory. At last, a case study is presented to show the concrete procedure of MSPER method.
\end{abstract}

\section{KEYWORDS}

Healthcare, Multiple Dimensions, Sport Physique Evaluation, Sport Score, TOPSIS, Weight

\section{INTRODUCTION}

With the continuous social progress and the gradual popularization of health idea, people are more and more interested in pursuing high-quality healthy life (Din \& Paul, 2020; Natalie, 2020). Generally, high-quality healthy life is often affected or influenced by sport physique of individuals. Typically, through monitoring, collecting, integrating and analyzing the sport scores or exercise scores (e.g., running score, swimming score, jumping score, and so on) of individuals, we can know about the sport physique of an individual in a real-time and efficient manner, so as to further maintain a highquality health level of people (Sillence et al., 2019; Szulc \& Duplaga, 2019).

However, in the abovementioned sport scores-based sport physique monitoring task, two major challenges or difficulties are raised. First of all, the collected sport scores for sport physique monitoring 
are often multi-dimensional (e.g., running score, swimming score, jumping score, and so on) (Dai et al., 2020; Kou et al., 2021; Wang et al., 2020; Zhong et al., 2020), which make it hard to evaluate the sport physique of individuals fairly and reasonably. Second, the multiple dimensions of sport scores (e.g., running score, swimming score, jumping score, and so on) are often of different weights or significances (Cai et al., 2018; Qi, Hu, Zhang et al, 2020), which raise a challenging task to evaluate the sport physique of an individual objectively and effectively.

Inspired by these challenges, a novel multi-dimensional sport physique evaluation and ranking method, named MSPER, is proposed in this research work for better healthcare for individuals, which is based on the TOPSIS theory in multi-objective decision problems. The major advantage of MSPER method is that it can overlook the complex weight design step that may place a heavy burden on the sport physique evaluation task, and hence can present an effective and objective sport physique evaluation result. At last, a case study is presented to show the concrete procedure of MSPER method.

In summary, our contributions in this paper are three-fold.

(1) We consider the multiple dimensions of sport scores (e.g., running score, swimming score, jumping score, and so on) of individuals when we evaluate and rank the candidate individuals according to the sport score values.

(2) We overlook the complex weight design step for the involved multiple sport score dimensions (e.g., running score, swimming score, jumping score, and so on) that may place a heavy burden on the sport physique evaluation task, and hence can present an effective and objective sport physique evaluation result.

(3) A case study is designed and presented to show the effectiveness and efficiency of the suggested MSPER method. Step-by-step case illustrations are introduced in detail to describe the MSPER method.

The paper structure is introduced in detail as follows. Paper motivation is presented in Section 2 through a real-world sport physique evaluation example where multiple dimensions of sport scores are involved. In Section 3, the details of the suggested MSPER method are clarified clearly step by step. A real-world case study for multi-dimensional sport physique evaluation and ranking tasks is presented in Section 4 to prove the effectiveness and efficiency of the MSPER method. At last, in Section 5, a conclusion is drawn and future research directions are discussed in detail to narrow the research focus in the future.

\section{MOTIVATION SPECIFICATION}

We use the example in Fig. 1 to describe the research background and significance of this work. In the example, there are three persons: Anna, Alice and Tom. Each person has three kinds of sport scores, i.e., \{running score, swimming score, jumping score , whose score values are present in Fig.1. Here, we assume that the score values of three persons are all known and available.

Moreover, the three sport scores should be of corresponding weights to indicate their respective importance degrees and significances in evaluating and ranking these three candidates: Anna, Alice and Tom, according to their respective sport scores. However, the weight design for different sport scores is often a non-trivial task as it may place a heavy burden on the objective sport physique evaluation of different candidate persons. In this situation, how to design an objective and fair sport physique evaluation method with multiple sport score dimensions but without weights is becoming a challenging issue that calls for continuous research. Inspired by this fact, a novel sport physique evaluation and ranking method, named MSPER, based on multi-dimensional sport scores is put forward in this paper for better healthcare services, which is basically based on the TOPSIS idea that is often used in multi-object optimization problems. The concrete steps are listed in the following section. 


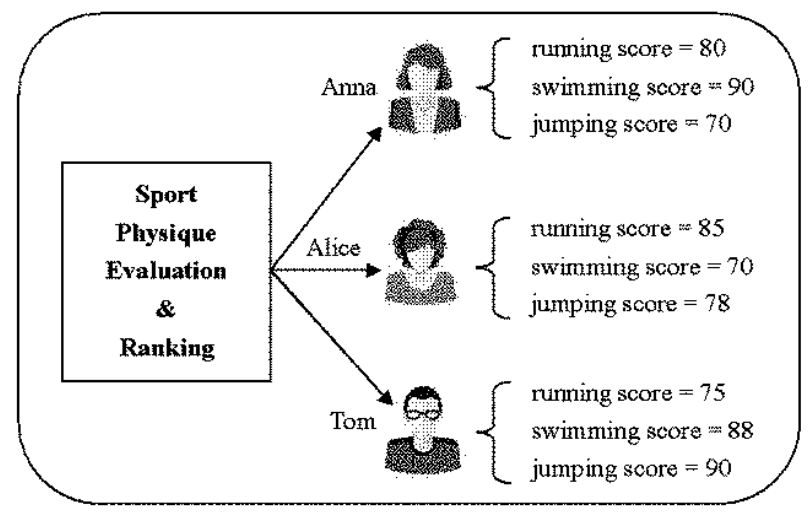

\section{METHOD}

In this section, we firstly introduce the concrete procedure of the suggested MSPER method briefly. Before the introduction, we first clarify the basic idea of TOPSIS when performing multi-dimensional data evaluation. The rationale of TOPSIS is: for a data point $\mathrm{P}$, if it is close to the best data point $\mathrm{X}_{1}$ but far away from the worst data point $\mathrm{X}_{2}$, then $\mathrm{P}$ is a good data point with high probability; Otherwise, if $\mathrm{P}$ is far away from the best data point $\mathrm{X}_{1}$ but close to the worst data point $\mathrm{X}_{2}$, then $\mathrm{P}$ is a bad data point with high probability.

The general idea of TOPSIS-based MSPER method is: (1) we first determine the best virtual person and the worst virtual person according to the multi-dimensional sport scores of all candidate persons (here, the word "virtual" means that the mentioned person is not definitely a real candidate person ready to be evaluated); (2) for each candidate person, we calculate his/ her

Step 1: Determining the best virtual person and the worst virtual person. According to the multidimensional sport scores of each candidate person $p_{i}$, we determine the best virtual person BP and the worst virtual person WP (in the form of multi-dimensional data points).

Step 2: Distances calculation for each candidate person. For each candidate person $p_{i}$, we calculate his/her distance Dist $\left(p_{i}, B P\right)$ with the best virtual person BP and his/her distance Dist $\left(p_{i}, W P\right)$ with the worst virtual person WP, respectively.

Step 3: Evaluation and ranking. We evaluate the comprehensive score Score $_{i}$ of each candidate person $p_{i}$ according to the two kinds of distances derived in Step 2. Afterwards, we rank all the candidate persons by their respective scores in descending order.

distance with the best virtual person and his/her distance with the worst virtual person, respectively; (3) for each candidate person, we evaluate his/her score based on the two kinds of distances obtained in (2) and finally, we rank all the candidate persons according to their respective scores. In summary, the three steps are presented in Fig.2.

For formal descriptions, we use $P=\left\{p_{1}, \ldots, p_{m}\right\}$ to denote the set of candidate persons, $S P=$ $\left\{s p_{1}, \ldots, s p_{n}\right\}$ to denote the set of sport items, $V=\left\{v_{i, j} \mid p_{i} \hat{\mathrm{I}} P, s p_{j} \hat{\mathrm{I}} S P\right\}$ to denote the set of scores of sport $s p_{j}$ by person $p_{i}$.

The relationship among the three steps of our proposal in Fig. 2 could be specified more intuitively by Fig.3. 
Figure 2. Three steps of MSPER

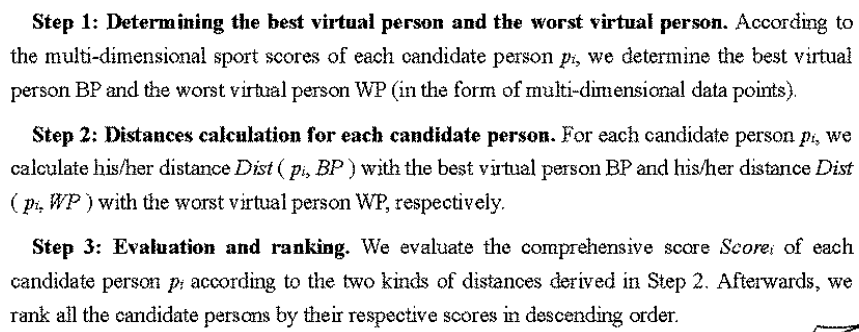

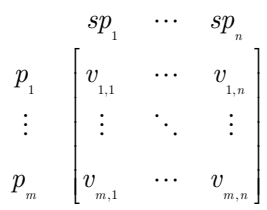

Step 1: Determining the best virtual person and the worst virtual person.

According to the person-sport scores of all the candidate persons, i.e., set $V=\left\{v_{i, j} \mid p_{i} \hat{\mathrm{I}} P, s p_{j}\right.$ $\hat{\mathrm{I} S P}$ \}, we can determine the best virtual person (abbreviated as $B P$ ) and the worst virtual person (abbreviated as $W P$ ). In concrete, for each sport form $s p_{j}$ in set $S P=\left\{s p_{1}, \ldots, s p_{n}\right\}$, we can get the maximal value (in the score evaluation scenario presented in Fig.1, sport score is of "the larger, the better" property) of $s p_{j}$ (denoted by $M a x_{j}$ ) and the minimal value of $s p_{j}$ (denoted by $\operatorname{Min}_{j}$ ). In this situation, we can construct the best virtual person, i.e., $B P$ and the worst virtual person, i.e., $W P$ with the formulas in equations (1)-(2). Here, as $n$ sports are present in set $S P$, both $B P$ and $W P$ are corresponding to $n$-dimensional vectors. As persons $B P$ and $W P$ are not definitely present in set $P=$ $\left\{p_{1}, \ldots, p_{m}\right\}$, we call $B P$ and $W P$ as best virtual person and worst virtual person, respectively.

$B P=\left(\operatorname{Max}_{1}, \ldots, \operatorname{Max}_{n}\right)$

$W P=\left(\operatorname{Min}_{1}, \ldots, \operatorname{Min}_{n}\right)$

Step 2: Distances calculation for each candidate person.

Next, for each candidate person $p_{i}$ in set $P=\left\{p_{1}, \ldots, p_{m}\right\}$, we calculate his or her distances with the best virtual person $B P$ and the worst virtual person $W P$, respectively.

First of all, we calculate the distance between the candidate person $p_{i}$ and the best virtual person BP. The distance is denoted by $\operatorname{Dist}\left(p_{i}, B P\right)$ and calculated by equation (3). Here, the distance calculation is based on Euclid Distance and generally, the smaller the Dist $\left(p_{i}, B P\right)$ is, the better the $p_{i}$ will be.

Dist $\left(p_{i}, B P\right)$

$$
=\operatorname{Dist}\left(\left(v_{i, 1}, \ldots, v_{i, n}\right),\left(\operatorname{Max}_{1}, \ldots, \operatorname{Max}_{n}\right)\right)
$$


$=\sqrt{\sum_{j=1}^{n}\left(v_{i, j}-M a x_{j}\right)^{2}}$

Likewise, we calculate the distance between the candidate person $p_{i}$ and the worst virtual person WP. The distance is denoted by $\operatorname{Dist}\left(p_{i}, W P\right)$ and calculated by equation (4). Here, the distance calculation is based on Euclid Distance and generally, the larger the Dist $\left(p_{i}, W P\right)$ is, the better the $p_{i}$ will be.

$$
\begin{aligned}
& \operatorname{Dist}\left(p_{i}, W P\right) \\
= & \operatorname{Dist}\left(\left(v_{i, 1}, \ldots, v_{i, n}\right),\left(\operatorname{Min}_{1}, \ldots, \operatorname{Min}_{n}\right)\right) \\
= & \sqrt{\sum_{j=1}^{n}\left(v_{i, j}-\operatorname{Min}_{j}\right)^{2}}
\end{aligned}
$$

This way, we have derived two distances for each candidate person $p_{i}(i=1,2, \ldots, m)$, i.e., $\operatorname{Dist}\left(p_{i}\right.$, $B P)$ (smaller is better) and $\operatorname{Dist}\left(p_{i}, W P\right.$ ) (larger is better). These two distances could be regarded as the evaluation basis for measuring the sport physique of the candidate persons based on their sport scores.

Step 3: Evaluation and ranking.

In Step 2, we have obtained two distances for each candidate person $p_{i}(i=1,2, \ldots, m)$, i.e., $\operatorname{Dist}\left(p_{i}, B P\right)$ (smaller is better) and $\operatorname{Dist}\left(p_{i}, W P\right.$ ) (larger is better). Next, in this step, we further integrate these two distances into one comprehensive score, so as to use the comprehensive score to measure or order the $m$ candidate persons in set $P=\left\{p_{1}, \ldots, p_{m}\right\}$ based on their respective sport scores and performances.

In concrete, for each candidate person $p_{i}(i=1,2, \ldots, m)$, his or her comprehensive score (denoted by Score $_{i}$ ) is calculated by equation (5). Here, the reason that we choose equation (5) for comprehensive score calculation is two-fold: $\operatorname{Dist}\left(p_{i}, B P\right)$ is of "smaller is better" type, $\operatorname{Dist}\left(p_{i}, W P\right)$ is of "larger is better" type. Thus, through (5), we can get the comprehensive score of each candidate person $p_{i}$, i.e., Score $_{i ;}$ moreover, for person $p_{i}$, $_{\text {core }}$ is of "larger is better" type. Then through Score ${ }_{i}$, we can evaluate the approximate sport physique of an individual $p_{i}$, and further rank all the candidate persons in descending order.

Score $_{i}=\frac{\operatorname{Dist}\left(p_{i}, W P\right)}{\operatorname{Dist}\left(p_{i}, B P\right)+\operatorname{Dist}\left(p_{i}, W P\right)}$

For more formalization, the pseudo code of the MSPER method is presented in Algorithm 1.

\section{Algorithm 1: MSPER}

Inputs:

(1) $P=\left\{p_{1}, \ldots, p_{m}\right\} / /$ candidate person set

(2) $S P=\left\{s p_{1}, \ldots, s p_{n}\right\} / /$ set of sport items

(3) $V=\left\{v_{i, j} \mid p_{i} \hat{\mathrm{I} P}, s p_{j} \hat{\mathrm{I}} S P\right\} / /$ person-sport scores

Output: 
Figure 3. Major Procedure of our proposed MSPER

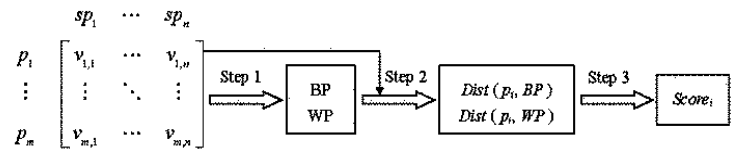

(1) $S C O R E=\left\{\right.$ Score $_{1}, \ldots$, Score $\left._{m}\right\} / /$ evaluation scores of $m$ candidate persons

1 For $j=1$ to $n$ do // Step 1: Determining BP and WP

$$
\begin{aligned}
& 2 \operatorname{Max}_{j}=0, \operatorname{Min}_{j}=¥ \\
& 3 \text { For } i=1 \text { to } m \text { do } \\
& 4 \text { If } v_{i, j}>\operatorname{Max}_{j}
\end{aligned}
$$

5 Then $\operatorname{Max}_{j}=v_{i, j}$

6 End If

7 If $v_{i, j}<\operatorname{Min}_{j}$

8 Then $\operatorname{Min}_{j}=v_{i, j}$

\section{End If}

\section{End For}

11 End For

$12 \mathrm{BP}=\left(\operatorname{Max}_{1}, \ldots, \operatorname{Max}_{n}\right) / /$ equation $(1)$

$13 \mathrm{WP}=\left(\operatorname{Min}_{1}, \ldots, \operatorname{Min}_{n}\right) / /$ equation (2)

14 For $i=1$ to $m$ do

15 calculate Dist $\left(p_{i}, B P\right)$ by (3) // Step 2: Distances calculation for $p_{i}$

16 calculate Dist $\left(p_{i}, W P\right)$ by (4)

17 calculate $S$ core ${ }_{i}$ by (5) // Step 3: Evaluation for $p_{i}$

18 put Score $_{i}$ into set SCORE

19 End For

\section{Return SCORE}

\section{CASE STUDY}

A case study is introduced in this section to prove the feasibility of the MSPER solution in this research work. The case study is constructed from the example in Fig.1, where there are three candidate persons: Anna, Alice and Tom and their scores on three kinds of sport items, i.e., $\{$ running, swimming, jumping \}. The concrete person-sport score values are present in Fig.1. Next, we should evaluate these three persons based on their respective sport score values without knowing the concrete weights of these three sport items beforehand. The concrete evaluation and ranking process is illustrated as follows.

(1) Step 1: Determining the best virtual person and the worst virtual person.

According to the sport scores of three candidate persons Anna, Alice and Tom, we can determine the best virtual person $B P$ and the worst virtual person $W P$. The concrete process is as equations (1)(2). For the three persons, their maximal scores on the three sport items, i.e., running, swimming, jumping are $85,90,90$, respectively. Likewise, their minimal scores on the three sport items, i.e., running, swimming, jumping are 75, 70, 70, respectively. Therefore, according to equations (1)-(2), we can get the following $B P$ and $W P$ : 


$$
\begin{aligned}
& B P=(85,90,90) \\
& W P=(75,70,70)
\end{aligned}
$$

(2) Step 2: Distances calculation for each candidate person.

The three persons Anna, Alice and Tom can be modeled as a three-dimensional point, respectively: Anna $(80,90,70)$, Alice $(85,70,78)$ and Tom $(75,88,90)$. Next, for each person, we calculate his or her distance with the best virtual person $B P$ and the worst virtual person $W P$, respectively. In concrete, the calculation process as shown as follows.

Dist $($ Anna, $B P)=$ Dist $((80,90,70),(85,90,90))=20.6$

Dist $($ Anna, $W P)=$ Dist $((80,90,70),(75,70,70))=20.6$

Dist $($ Alice, $B P)=$ Dist $((85,70,78),(85,90,90))=23.3$

Dist $($ Alice, $W P)=$ Dist $((85,70,78),(75,70,70))=12.8$

Dist $($ Tom, $B P)=$ Dist $((75,88,90),(85,90,90))=10.2$

Dist $($ Tom, $W P)=$ Dist $((75,88,90),(75,70,70))=26.9$

\section{(3) Step 3: Evaluation and ranking.}

For each candidate person, we can calculate his or her comprehensive score according to the equation (5). In concrete, the calculation process is detailed as follows.

$$
\begin{aligned}
& \text { Score }_{\text {Anna }}=20.6 /(20.6+20.6)=0.5 \\
& \text { Score }_{\text {Alice }}=12.8 /(23.3+12.8)=0.355 \\
& \text { Score }_{\text {Tom }}=26.9 /(10.2+26.9)=0.725
\end{aligned}
$$

The comprehensive score values are of "the larger, the better" type; therefore, among the three candidate persons, Tom is the best in terms of sport physique and Alice is the worst in terms of sport physique.

\section{CONCLUSION}

Sport physique monitoring and evaluation are crucial to the healthcare of whole nation and populations. Sport item scores have provided a promising way to objectively evaluate the sport physique of individuals of the nation. However, abovementioned sport score-based sport physique monitoring methods often challenge in multiple dimensions of sport scores as well as each dimension's weight value which is often hard to design or capture. Considering the above two challenges, we put forward a novel multi-dimensional sport physique evaluation and ranking method, named MSPER, in this research work for better healthcare for individuals, which is based on the TOPSIS theory. At last, a concrete case study is presented to show the detailed procedure of the MSPER method.

In the future work, we intend to further improve the MSPER method by considering more data formats (Cai \& He, 2019; He et al., 2017; Liu et al., 2020; Xian et al., 2018). In addition, how to secure the sensitive user information (Cai \& Zheng, 2020; Liu, Hou, Wang et al, 2019; Liu, Tian, Wu et al, 2019; Qi, Wang, Xu et al, 2020; Sun et al., 2021; Xiao et al., 2017) is another interesting topic that requires continuous investigation. 


\section{REFERENCES}

Cai, Z., \& He, Z. (2019). Trading private range counting over big IoT data. The 39th IEEE International Conference on Distributed Computing Systems (ICDCS 2019). doi:10.1109/ICDCS.2019.00023

Cai, Z., He, Z., Guan, X., \& Li, Y. (2018). Collective data-sanitization for preventing sensitive information inference attacks in social networks. IEEE Transactions on Dependable and Secure Computing, 15(4), 577-590.

Cai, Z., \& Zheng, X. (2020). A private and efficient mechanism for data uploading in smart cyber-physical systems. IEEE Transactions on Network Science and Engineering, 7(2), 766-775. doi:10.1109/TNSE.2018.2830307

Dai, H., Ji, Y., Liu, L., Yang, G., \& Yi, X. (2020). A privacy-preserving multi-keyword ranked search over encrypted data in hybrid clouds. IEEE Access: Practical Innovations, Open Solutions, 8, 4895-4907. doi:10.1109/ ACCESS.2019.2963096

Din, S., \& Paul, A. (2020). Smart health monitoring and management system: Toward autonomous wearable sensing for Internet of Things using big data analytics. Future Generation Computer Systems, 111, 939-939. doi:10.1016/j.future.2019.06.034

He, Q., Zhou, R., Zhang, X., Wang, Y., Ye, D., Chen, F., Grundy, J., \& Yang, Y. (2017). Keyword search for building service-based systems. IEEE Transactions on Software Engineering, 43(7), 658-674. doi:10.1109/ TSE.2016.2624293

Kou, H., Liu, H., Duan, Y., Gong, W., Xu, Y., Xu, X., \& Qi, L. (2021). Building trust/distrust relationships on signed social network through privacy-aware link prediction. Applied Soft Computing, 100, 106942. Advance online publication. doi:10.1016/j.asoc.2020.106942

Liu, Kou, Yan, \& Qi. (2020). Keywords-Driven and Popularity-Aware Paper Recommendation Based on Undirected Paper Citation Graph. Complexity. doi: 10.1155/2020/2085638

Liu, Q., Hou, P., Wang, G., Peng, T., \& Zhang, S. (2019). Intelligent route planning on large road networks with efficiency and privacy. Journal of Parallel and Distributed Computing, 2019(133), 93-106. doi:10.1016/j. jpdc.2019.06.012

Liu, Q., Tian, Y., Wu, J., Peng, T., \& Wang, G. (2019). Enabling verifiable and dynamic ranked search over outsourced data. IEEE Transactions on Services Computing, 1. Advance online publication. doi:10.1109/ TSC.2019.2922177

Natalie, C. (2020). Broadband Internet access is a social determinant of health! American Journal of Public Health, 110(8), 1123-1125. doi:10.2105/AJPH.2020.305784 PMID:32639914

Qi, L., Hu, C., Zhang, X., Khosravi, M. R., Sharma, S., Pang, S., \& Wang, T. (2020). Privacy-aware data fusion and prediction with spatial-temporal context for smart city industrial environment. IEEE Transactions on Industrial Informatics. Advance online publication. doi:10.1109/TII.2020.3012157

Qi, L., Wang, X., Xu, X., Dou, W., \& Li, S. (2020). Privacy-aware cross-platform service recommendation based on enhanced locality-sensitive hashing. IEEE Transactions on Network Science and Engineering. Advance online publication. doi:10.1109/TNSE.2020.2969489

Sillence, E., Blythe, J. M., Briggs, P., \& Moss, M. (2019). A revised model of trust in internet-based health information and advice: Cross-sectional questionnaire study. Journal of Medical Internet Research, 21(11), e11125. doi:10.2196/11125 PMID:31710297

Sun, Z., Wang, Y., Cai, Z., Liu, T., Tong, X., \& Jiang, N. (2021). A two-stage privacy protection mechanism based on blockchain in mobile crowdsourcing. International Journal of Intelligent Systems, 36(5), 2058-2080. Advance online publication. doi:10.1002/int.22371

Szulc, K., \& Duplaga, M. (2019). The impact of Internet use on mental wellbeing and health behaviours among persons with disability. European Journal of Public Health, 29(4), ckz185.425. Advance online publication. doi:10.1093/eurpub/ckz185.425

Wang, Y., Gao, Y., Li, Y., \& Tong, X. (2020). A worker-selection incentive mechanism for optimizing platformcentric mobile crowdsourcing systems. Computer Networks, 107, 107144. doi:10.1016/j.comnet.2020.107144 
Xian, Z., Li, Q., Huang, X., Lu, J., \& Li, L. (2018). Differential privacy protection for collaborative filtering algorithms with explicit and implicit trust. Tien Tzu Hsueh Pao, 46(12), 3050-3059.

Xiao, Y., Xiong, L., Zhang, S., \& Cao, Y. (2017). LocLok: Location cloaking with differential privacy via hidden Markov model. Proceedings of the VLDB Endowment International Conference on Very Large Data Bases, 10(12), 1901-1904. doi:10.14778/3137765.3137804

Zhong, W., Yin, X., Zhang, X., Li, S., Dou, W., Wang, R., \& Qi, L. (2020). Multi-dimensional quality-driven service recommendation with privacy-preservation in mobile edge environment. Computer Communications, 157, 116-123. doi:10.1016/j.comcom.2020.04.018

Changqing Zhuo received his bachelor degree in 2002 from the Physical Education Department of Chengdu Institute of Physical Education, China, majoring in Physical Education. He has been working in the Engineering \& Technical College of Chengdu University of Technology since 2012 as the director of the college sport research and teaching section of the Department of Physical Education. His research interests include Physical Education and Sports Training.

Qiyun Zhang studied in Anshan Normal College from 1993 to 1995, majoring in physical education and training. Since graduation, he has been working in the front line of physical education, during which he obtained a master degree in physical education from Hebei Normal University in 2002. Since 2002, he began to coach the school track and field training team, and accumulated a wealth of teaching and training experience. Over the years, he has published many high-quality articles in various academic journals. His research interest is physical education and training. He is currently an associate professor of Weifang Key Laboratory of Blockchain on Agricultural Vegetables, Weifang University of Science and Technology, China

Qin Xie received her bachelor degree from the Physical Education Department of Chengdu Sport University, China, in 2002 and 2016, respectively. Since 2002, she has been teaching in Sichuan Staff University of Science and Technology, China. Her research interest is physical education and training.

Yinping Lin received her bachelor degree and master degree from the Sports Science College of Qufu Normal University, China, in 2004 and 2008, respectively. Her majority is physical education. Currently, she is teaching in No.1 Middle School of Pidu Chengdu, China. Her research interest is physical education and training.

Chengxin Xu received his bachelor degree from School of Information Science and Engineering, Qufu Normal University, China, in 2019. Now, he is pursuing his master degree at the School of Computer Science, Qufu Normal University, China. His research interests are big data and artificial intelligence.

Lina Wang received her bachelor degree from School of Software, Qufu Normal University, China, in 2019. Now, she is pursuing her master degree at the School of Computer Science, Qufu Normal University, China. Her research interests are big data and recommender systems.

Sifeng Wang received his bachelor and master degree from School of Mathematics and Computer Science, Qufu Normal University, China, in 1996 and 2011, respectively. Now, he is an engineering at the School of Computer Science, Qufu Normal University, China. His research interests are big data and artificial intelligence. He is the corresponding author of this paper. 